\title{
Improving the Spatial Resolution of Atomic-Scale EDS Mapping for Chemical Imaging and Quantification of Metallic Alloy Structures
}

\author{
Ping Lu ${ }^{1}$, Eric Romero ${ }^{1}$, Lin Zhou $^{2}$, M. J. Kramer ${ }^{2}$, and David J. Smith ${ }^{3}$ \\ ${ }^{1}$ Sandia National Laboratories, PO Box 5800, MS 1411, Albuquerque, NM 87185-1411 USA \\ ${ }^{2}$ Ames Laboratory, Ames, IA 50014 USA \\ ${ }^{3}$ Department of Physics, Arizona State University, Tempe, AZ 85287 USA
}

Recent technical advances in scanning transmission electron microscopy (STEM) and x-ray detector technology have made it possible to do atomic-resolution chemical mapping using energydispersive x-ray spectroscopy (EDS). So far, most of these efforts have been applied to materials such as perovskite oxides [1-3], e.g. $\mathrm{SrTiO}_{3}$, and compound semiconductors [4, 5], which have well-known crystal structures with relatively large lattice spacings, that are also comparatively resistant to electronbeam irradiation. This radiation-resistant property allows longer dwell times at each recording point (i.e., pixel) for achieving the signal-to-noise $(\mathrm{S} / \mathrm{N})$ ratio that is necessary for elemental mapping. For the study of intermetallics such as body-centered-cubic (bcc) alloys that have small interatomic distances and are relatively sensitive to the electron radiation, improvement in the spatial resolution as well as reduction in the overall electron dose are required. In this work, we report our strategy to overcome these difficulties as well as applications of the techniques for quantification of chemical composition at the lattice sites in intermetallic bcc alloys [6].

In chemical mapping, the shortest lateral distance between columns of identical atoms in a crystal projection determines the required spatial resolution necessary for resolving the atomic columns $[6,7]$. The required spatial resolution is $3.9 \AA$ for $\mathrm{SrTiO}_{3}$ in the [001] direction and about $3.5 \AA$ for GaAs in the [110] direction. The smaller dimensions of the unit cell $(\sim 2.9 \AA)$ for bcc intermetallic alloys require higher spatial resolution. This improvement can be achieved by a combination of small electron probe $(<1.5 \AA)$, using a thin TEM specimen $(<20 \mathrm{~nm})$ and improving the $\mathrm{S} / \mathrm{N}$ ratio through averaging of EDS maps that are related to each other via lattice-vector translations in the image plane (for convenience, we have termed this process "lattice-averaging"). Significant improvement in the S/N ratio of EDS mapping can be achieved by lattice-averaging (Fig.1), which compensates for reduction in X-ray count due to the selection of small electron probe and thin specimen, and also leads to an overall reduction of electron dose necessary for EDS mapping [6]. By choosing a thin TEM specimen, the EDS signal is localized to atomic columns $[1,3,6,7]$ and $\mathrm{X}$-ray counts from individual atomic columns can be approximated by a Gaussian distribution. The width of the Gaussian peak is then effectively determined by convoluting the electron probe with the effective EDS local ionization potential, leading to a spatial resolution of better than $2.0 \AA$ [6]. Fig. 2 shows EDS maps of Fe $\mathrm{K} \alpha$ and Co Ka, obtained from a Fe-Co alloy, showing an ordered bcc structure with the $\mathrm{Fe}$ and Co atoms preferentially occupying the A-sites and B-sites in the bcc lattice [6]. Furthermore, the chemical composition at the atomic column positions can be quantified column-by-column using the Cliff-Lorimer method [6-9].

\section{References}

1. A.J. D’Alfonso, B. Freitag, V. Klenov \& L.J. Allen Phys. Rev. B 81, 100101 (2010).

2. L.J. Allen, A.J. D’Alfonso., B. Freitag, \& D.O. Klenov,. MRS Bulletin 37, 47 (2012).

3. P. Lu, J. Xiong, M. Van Benthem . \& Q.X. Jia App. Phys. Lett. 102, 173111 (2013).

4. M.W.Chu, S.C.Liou, C.P. Chang, F.S.Choa \& C.H.Chen. Phys, Rev.Lett. 104,196101(2010).

5. D.O. Klenov, \& J. M. O. Zide, App. Phys. Lett. 99, 141904 (2011). 
6. P. Lu, L. Zhou, M.J. Kramer \& D. J. Smith, Sci. Rep. 4, 3945 (2014)

7. P. Lu, E. Romero, S. Lee, J. L. MacManus-Driscoll \& Q. X. Jia, Submitted to Microscopy and Microanalysis, (2014).

8. G. Cliff \& G.W. Lorimer, J. Microsc. 103, 203-207 (1975).

9. Sandia National Laboratories is a multi-program laboratory managed and operated by Sandia Corporation, a wholly owned subsidiary of Lockheed Martin Corporation, for the US Department of Energy's National Nuclear Security Administration under contract DE-AC04-94AL85000. The work at Ames Laboratory was supported by the Department of Energy-Energy Efficiency and Renewable Energy, Vehicles Technology Office, PEEM program, under Contract No. DE-AC02-07CH11358 for the operation of Ames Laboratory (USDOE).
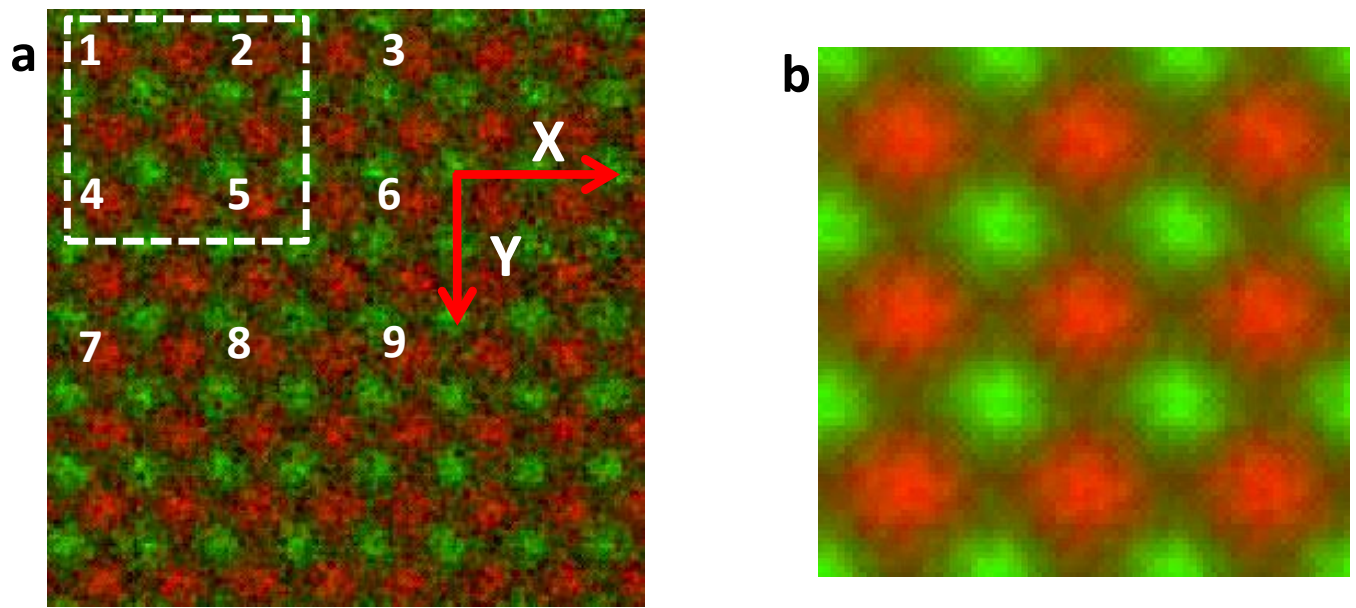

Figure 1. Method of the lattice averaging used to improve S/N ratio of EDS mapping: (a) a raw EDS map; and (b) lattice-averaged EDS map. In (a), a smaller EDS region, size of 3x3 unit cells (marked by dashed square) is chosen, maps of this region from nine equivalent positions (marked by $1,2, \ldots 9)$ in the image, defined by using basic lattice translations vectors $(\mathbf{X}, \mathbf{Y})$, are averaged to obtain the lattice-averaged map in (b). In this example, $\mathbf{X}=2 a_{0} \overrightarrow{\boldsymbol{x}}$, and $\mathbf{Y}=2 a_{0} \overrightarrow{\boldsymbol{y}}$ are chosen as basic translation vectors, where $\mathrm{a}_{\mathrm{o}}$ is the unit cell constant and $\overrightarrow{\boldsymbol{x}}$ and $\overrightarrow{\boldsymbol{y}}$ are the unit vectors in $\mathrm{x}-$, and $\mathrm{y}$ - directions.
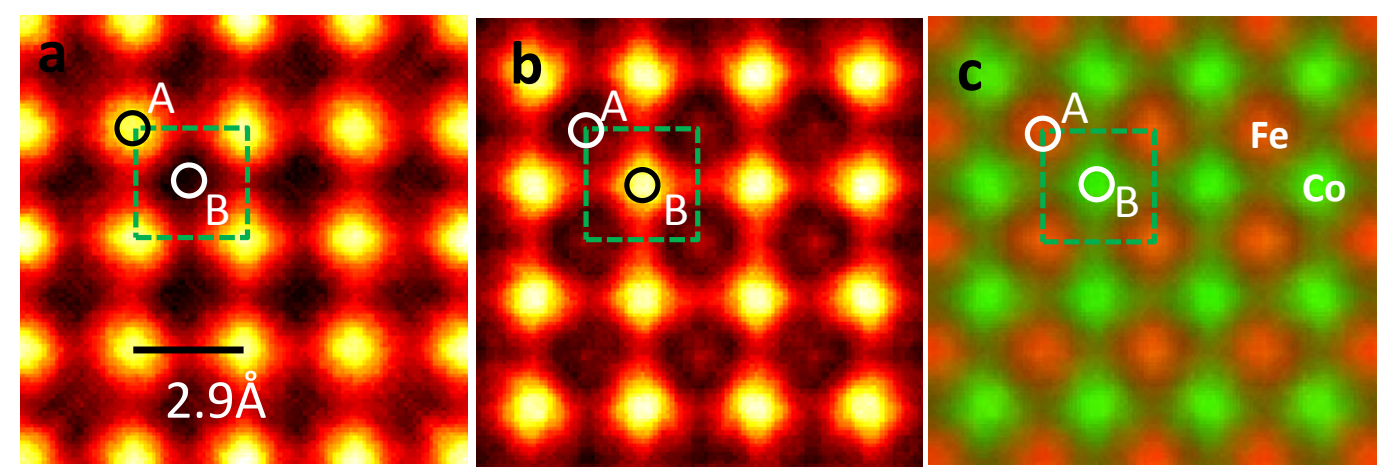

Figure 2. EDS map of (a) $\mathrm{Fe} \mathrm{K}_{\alpha}$; (b) $\mathrm{Co} \mathrm{K}_{\alpha}$; and (c) composite color map of Fe $\mathrm{K}_{\alpha}$ (red) and Co $\mathrm{K}_{\alpha}$ (green), obtained from a Fe-Co alloy. Lattice site positions (A, B) and size of unit cell in [001] projection are marked by circles and dashed square in EDS maps in (a), (b) and (c). 